\title{
Formal derivation of dissipative particle dynamics from first principles
}

\author{
David Cubero* $^{*}$ and Sophia N. Yaliraki ${ }^{\dagger}$ \\ Department of Chemistry, South Kensington Campus, Imperial College London, London SW7 2AZ, United Kingdom
}

(Received 13 January 2005; revised manuscript received 20 May 2005; published 13 September 2005)

\begin{abstract}
We show that the Markovian approximation assumed in current particle-based coarse-grained techniques, like dissipative particle dynamics, is unreliable in situations in which sound plays an important role. As an example we solve analytically and numerically the dynamics of coarse-grained harmonic systems by using first principle methods, showing the presence of long-lived memory kernels. This effect raises questions about the connection of these approaches at their current form to molecular dynamics.
\end{abstract}

DOI: 10.1103/PhysRevE.72.032101

PACS number(s): 05.40.-a, 02.70.Ns

The processes underlying the behavior of large physical and biological macromolecular assemblies span a range of length and time scales that often require coupling of microscopic interactions with continuum-level properties that cannot be presently bridged by techniques like molecular dynamics. This has recently led to revisiting existing or developing new coarse-graining schemes. Particle-based methods [1-5] based on the dissipative particle dynamics (DPD) methodology have been used extensively in recent years as models for addressing such systems at the mesoscopic level $[6,7]$. One of the appeals of DPD is the capability of reproducing hydrodynamic behavior through the conservative, dissipative, and stochastic pairwise forces that are mass and momentum conserving. Manifestations of the continuum properties at the mesoscale have been extensively investigated. Nevertheless, this methodology is still lacking a clear connection with the underlying microscopic level. In Ref. [4] a derivation of DPD from the microscopic level was proposed. However, this derivation was based on a series of ad hoc assumptions about the form of the conservative, dissipative, and noise forces. In particular, it was assumed that the fluctuating forces are Markovian. A more fundamental approach was presented in Ref. [5], in which the equation of motion for the dissipative particles was obtained by means of projection operators. As a result, the dissipative forces present memory kernels and the fluctuating forces are coupled to the dissipative terms via the fluctuationdissipation theorem. However, the DPD-like equations of motion are only obtained when it is assumed that the fluctuating forces are Markovian and the memory kernels collapse into the familiar dissipative forms, functions only of the instantaneous values of the coarse-grained variables. In Ref. [5], this was justified by arguing that the coarse-grained variables are mesoscopic, and therefore slow when compared with the microscopic variables. Since this was a central requirement for obtaining the equations of motion, it merits verification.

In this work we show that the Markovian approximation is not applicable in situations in which sound plays an im-

\footnotetext{
* Present address: Física Teórica, Universidad de Sevilla, Apartado de Correos 1065, Sevilla 41080, Spain.

†Electronic address: s.yaliraki@imperial.ac.uk
}

portant role. We first study the general case and the conditions for the failure of the Markovian approximation due to sound propagation. Then, as an example, we obtain the memory kernels of the coarse-grained dynamics of cubic harmonic lattices in several dimensions by using first principle methods. Harmonic lattices are paradigmatic for the kind of memory effect discussed here because elastic wave propagation is the only dissipation mechanism in these systems. We have recently used projector operators to formally derive the stochastic equations of motion for inhomogeneous harmonic lattices [8]. We use these results as a starting point in order to evaluate here for the first time the required memory kernels, analytically in one dimension and numerically in two and three dimensions.

Consider a coarse-grained harmonic system by partitioning the volume into mesoscopic regions $k$. We define the coarse-grained particles by choosing the set of all center of mass coordinates $X_{k}$ and velocities $V_{k}$ of all particles inside each region $k$ as the relevant variables. Then, by using Mori's projection operator theory [9], the following equation of motion for the coarse-grained variables is obtained [8]:

$$
M_{k} \frac{d V_{k}(t)}{d t}=-\sum_{l}\left(\Lambda_{k l} X_{l}(t)+\int_{0}^{t} d \tau \Phi_{k l}(\tau) V_{l}(t-\tau)\right)+R_{k}(t)
$$

where $M_{k}$ is the mass of each coarse-grained particle; $\Lambda_{k l}$ is a conservative force matrix, $\Phi_{k l}(t)$ is a memory kernel defining dissipation; and $R_{k}(t)$ is a random force satisfying $\left\langle R_{k}(t)\right\rangle=0$ and the fluctuation-dissipation theorem $\left\langle R_{k}(t) R_{l}(0)\right\rangle=k_{B} T \Phi_{k l}(t)$, where $k_{B}$ is the Boltzmann constant and $T$ is the temperature. In these last equations the averages are taken with an initial distribution close to thermal equilibrium. Total momentum conservation implies that $\sum_{l} \Phi_{k l}(t)$ $=\Sigma_{l} R_{l}(t)=0$, so that if we decompose each random force as a sum of independent variables $R_{k}(t)=\Sigma_{l \neq k} \xi_{k l}$, we can rewrite (1) in a form that resembles the equation of motion of a DPD system, 


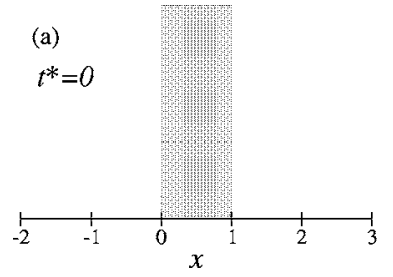

(b)

(c)

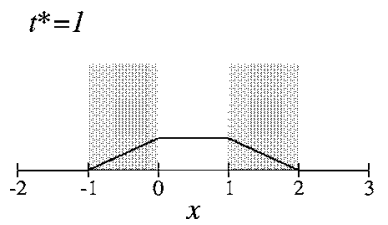

(d)

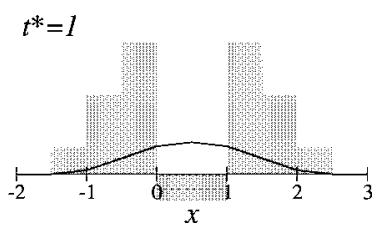

FIG. 1. Time evolution of the profiles of $u(x, t)$ (solid line) and $\partial u / \partial t(x, t)$ (shaded region) in a one-dimensional system: (a) initial condition, (b) after a time $\tau_{s} / 2$, (c) after a time $\tau_{s}$, (d) after a time evolution $\tau_{s} / 2$ from (b), but with a different profile, $\partial u / \partial t$, given by the dashed line in (b).

$$
\begin{aligned}
M_{k} \frac{d V_{k}(t)}{d t}= & -\sum_{l \neq k}\left\{\Lambda_{k l}\left[X_{l}(t)-X_{k}(t)\right]-\xi_{k l}(t)\right. \\
& \left.+\int_{0}^{t} d \tau \Phi_{k l}(t-\tau)\left[V_{l}(\tau)-V_{k}(\tau)\right]\right\} .
\end{aligned}
$$

However, only if the kernels are Dirac delta functions we obtain the DPD equation of motion. This would imply that the set of coarse-grained variables $\left\{X_{k}, V_{k}\right\}$ is Markovian.

To show that this is not the case when sound propagation is considered, let us focus on a simple one-dimensional solid. At the macroscopic level, the displacement field $u(x, t)$ [10] is defined as the deviation from equilibrium of the particles whose equilibrium site is $x$. If we neglect the microscopic fluctuations, we can express the mesoscopic variables as $X_{k}(t)=\left\langle X_{k}\right\rangle_{\mathrm{eq}}+\int_{l k}^{l(k+1)} d x u(x, t)$ and $V_{k}(t)$ $=\int_{l k}^{l(k+1)} d x \partial u(x, t) / \partial t$, where $l$ is the size of the coarsegrained regions and $\langle\cdots\rangle_{\text {eq }}$ denotes equilibrium averages. In the simplest case the displacement field verifies the wave equation $\partial^{2} u / \partial t^{2}=v^{2} \partial^{2} u / \partial x^{2}$, where $v$ is the speed of sound in the material. The general solution to this equation is given by $u(x, t)=f(x-v t)+g(x+v t)$, with a front $f(x)$ and $g(x)$ traveling to right and left, respectively. Note that the ratio $\tau_{s}$ $=l / v$ defines a mesoscopic time scale because $l$ is mesoscopic. Now consider that initially all coarse-grained particles are in their equilibrium positions with zero velocity except one, which we label as $k=0$. In Fig. 1(a) we have plotted the corresponding fields $u(x, 0)$ (solid line) and $(\partial u / \partial t)(x, 0)$ (shaded area). Assume that the fluctuations are small enough so that the fronts are not destroyed. Then, Figs. 1(b) and 1(c) show the profiles after a time $\tau_{s} / 2$ and $\tau_{s}$, respectively. Now imagine that at the intermediate time $t=\tau_{s} / 2$ we change the velocity field $\partial u / \partial t$ so that the set of coarse-grained variables $\left\{X_{k}, V_{k}\right\}$ remains the same, as shown in Fig. 1(b) (dashed line). Figure $1(\mathrm{~d})$ shows the profiles after a time $\tau_{s} / 2$. We can clearly see that the values of the coarse-grained variables are very different from the former case shown in Fig. 1(c), even though they had the same values at $t=\tau_{s} / 2$. Therefore, the knowledge of the values at a previous instant is not sufficient. Instead, we need to know the complete profiles of $u$ and $\partial u / \partial t$ at a given time or, equivalently, the complete previous history of $\left\{X_{k}, V_{k}\right\}$ to specify the present state.

The arising memory kernels are the consequence of the perturbations traveling inside the coarse-grained particles by sound propagation. This phenomenon is due to the space discretization introduced by the coarse graining and we expect it to be present even if we consider fluctuations, higher dimensions, or take into account anharmonic effects, as long as the mesoscopic time scale associated with sound propagation is comparable with the relevant time scale of the mesoscopic particles. If $\tau_{s}$ is much shorter than other characteristic times associated with distinct dissipation mechanisms present in the system, then the memory kernels due to sound propagation would collapse in the longer time scales and the previous discussion no longer applies. However, in situations in which sound propagation plays an important role, the coarse-grained variables must change in the same scale given by $\tau_{s}$ in order to account properly for it, which implies the effect described above.

As an example, let us consider a $d$-dimensional cubic harmonic lattice with only first neighbor interactions. Units are defined so that the mass of each oscillator, the force constants, and the equilibrium spacings are set to unity. We coarse-grain the system by grouping the oscillators into cubic clumps, each containing $n^{d}$ oscillators. It was shown in Ref. [8] that the conservative force $\Lambda_{k l}$ is the inverse of the matrix $\left\langle X_{k} X_{l}\right\rangle_{\mathrm{eq}} / k_{B} T$. In harmonic systems, the mesoscopic time scale that characterizes the dynamics of the coarsegrained particles is given by wave propagation only, which in our units is $\tau_{s}=n$. Therefore, by changing to $t^{*}=t / n$, with $V_{k}^{*}=d X_{k} / d t^{*}=n V_{k}$, Eq. (1) becomes

$$
\frac{d V_{k}^{*}}{d t^{*}}=-\sum_{l}\left(\Lambda_{k l}^{*} X_{l}+\int_{0}^{t^{*}} d \tau \Phi_{k l}^{*}(\tau) V_{l}^{*}\left(t^{*}-\tau\right)\right)+R_{k}^{*}
$$

where $\Lambda_{k l}^{*}=n^{2-d} \Lambda_{k l}, \Phi_{k l}^{*}=n^{2-d} \Phi_{k l}$, and $R_{k}^{*}=n^{2-d} R_{k}$. Since we have set all internal parameters to unity, we expect that all variables in Eq. (3), and, in particular, $\Lambda_{k l}^{*}$ and $\Phi_{k l}^{*}$, are on the order of $O(1)$, even in the mesoscopic limit $n \rightarrow \infty$. The analytic and simulation results we present later confirm this scaling in all three relevant physical dimensions, i.e., $d=1,2$, and 3 .

To continue analytically, we restrict ourselves temporarily to the one-dimensional (1-D) case. Let us consider a finite chain of $N$ oscillators with fixed ends and then take the thermodynamic limit $(N \rightarrow \infty)$ at the end of the calculations. The force matrix for the oscillators in this system is given by $A_{i j}=2 \delta_{i j}-1\left(\delta_{i, j+1}+\delta_{i, j-1}\right)$. Using the analytical expression for the inverse matrix $A_{i j}^{-1}=\min (i, j)[1-\max (i, j) /(N+1)][11]$, we obtain

$$
\begin{aligned}
\frac{\left\langle X_{k} X_{l}\right\rangle_{\mathrm{eq}}}{k_{B} T}= & -\frac{n^{2}-1}{6 n} \delta_{k l}+\left(\frac{[1+2 \min (k, l) n-n]}{2}\right) \\
& \times\left(1-\frac{1+2 \max (k, l) n-n}{2(N+1)}\right) .
\end{aligned}
$$

Next, we invert this matrix in two steps. First we multiply it 
by $A$, which leads to a tri-diagonal matrix $S_{k l}$ $\equiv \Sigma_{l^{\prime}}\left\langle X_{k} X_{l^{\prime}}\right\rangle_{\mathrm{eq}} A_{l^{\prime} l^{\prime}} / k_{B} T=a \delta_{i j}+b\left(\delta_{i, j+1}+\delta_{i, j-1}\right)$, with $a=1 / 3 n$ $+2 n / 3$ and $b=\left(n^{2}-1\right) / 6 n$. Strictly speaking, the first and last columns of $S$ do not follow that tri-diagonal form, but this is irrelevant in the thermodynamic limit. Now we invert the matrix $S$ by using the form $S_{k l}^{-1}=C \nu^{|k-l|}$, which leads to $C$ $=1 / \sqrt{a^{2}-4 b^{2}}$ and $\nu=-\left(a / b-\sqrt{\left.(a / b)^{2}-4\right)} / 2\right.$. The fact that $a / b>2$ for any $n$ guarantees that $|\nu|<1$ and consequently $S^{-1}$ vanishes at the boundaries. The force matrix is then given by $\Lambda=A \times S^{-1}$, resulting in

$$
\begin{aligned}
\Lambda_{k l}= & \sqrt{\frac{3}{2+n^{2}}}\left(\frac{6 n^{2}}{n^{2}-1}-\frac{2 \sqrt{3} n \sqrt{2+n^{2}}}{n^{2}-1} \delta_{k l}\right) \\
& \times(-1)^{|k-l|}\left(\frac{1+2 n^{2}-\sqrt{3} n \sqrt{2+n^{2}}}{n^{2}-1}\right)^{|k-l|} .
\end{aligned}
$$

In the mesoscopic limit $(n \rightarrow \infty)$, it reduces to [14]

$$
\Lambda_{k l} \sim \frac{2 \sqrt{3}}{n}\left(3-\sqrt{3} \delta_{k l}\right)(-1)^{|k-l|}(2-\sqrt{3})^{|k-l|} .
$$

Note that since $|2-\sqrt{3}|<1$, Eq. (6) predicts an exponential decay of the conservative force, in contrast with the standard linear decay used in DPD simulations [2].

Let us now turn our attention to the memory kernels. The autocorrelation matrix $C_{k l}^{*}\left(t^{*}\right)=\left\langle V_{k}^{*}\left(t^{*}\right) V_{l}^{*}(0)\right\rangle_{\mathrm{eq}}$ satisfies the same equation as $V_{k}^{*}$, Eq. (3), but without the random force [9]. Taking the Laplace transforms $\phi(s)$ $=\int_{0}^{\infty} d t^{*} \exp \left(-s t^{*}\right) \Phi^{*}\left(t^{*}\right), c(s)=\int_{0}^{\infty} d t^{*} \exp \left(-s t^{*}\right) C^{*}\left(t^{*}\right)$, we arrive at

$$
\sum_{l^{\prime}} \phi_{k l^{\prime}}(s) c_{l^{\prime} l}(s)=C_{k l}^{*}(0)-s c_{k l}(s)-\sum_{l^{\prime}} \frac{\Lambda_{k l^{\prime}}^{*}}{s} c_{l^{\prime} l}(s),
$$

where $C_{k l}^{*}(0)=\delta_{k l} n^{2} k_{B} T / M_{l}$. The autocorrelation matrix can also be readily calculated in the mesoscopic limit using the macroscopic wave equation [8], leading to

$$
C_{k l}^{*}\left(t^{*}\right)=C_{l l}^{*}(0)\left(1+\delta_{k l}\right) g\left(t^{*}-|k-l|\right) / 2,
$$

where $g(x)=(1-|x|) H(1-|x|)$ and $H(x)$ is the Heaviside unit step function. Therefore, we can compute the kernels by inverting the autocorrelation matrix

$$
\left.c_{k l}(s)=\frac{C_{l l}^{*}(0)}{s^{2}}\left(\delta_{k l}\left(e^{-s}+s-1\right)+e^{-s(1+\mid k-l)}\right) \frac{\left(e^{s}-1\right)^{2}}{2}\left(1-\delta_{k l}\right)\right) .
$$

We do that in two steps. First we multiply $c$ by the intermediate matrix $R_{k l}=\cosh (s) \delta_{k l}-(1 / 2)\left(\delta_{k, l+1}+\delta_{k, l-1}\right)$. The resulting matrix is tridiagonal and can be inverted using the same method we applied for the conservative force. The final result for the memory kernel is

$$
\begin{aligned}
\phi_{k l}(s)= & \frac{s^{2}[-\xi(s)]^{k-l \mid}}{[s-\sinh (s)] \mu(s)}\left[\sinh (s)[1-\cosh (s)]+\delta_{k l} \mu(s)\right] \\
& -s \delta_{k l}-\frac{\Lambda_{k l}^{*}}{s}
\end{aligned}
$$

where

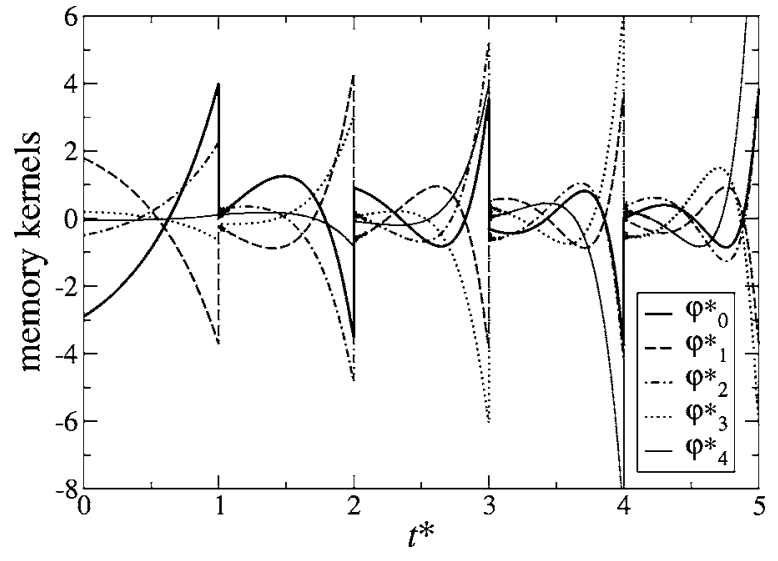

FIG. 2. Memory kernels $\varphi_{k l}^{*}\left(t^{*}\right)$ in one dimension.

$$
\xi(s)=\frac{\sinh (s)-s \cosh (s)+\mu(s)}{s-\sinh (s)},
$$

and

$$
\mu(s)=\sqrt{s[\cosh (s)-1][s+s \cosh (s)-2 \sinh (s)]} .
$$

The first piece of information we can extract from (10) is the friction coefficient that would be used in a DPD theory,

$$
\int_{0}^{\infty} d t^{*} \Phi_{k l}^{*}\left(t^{*}\right)=\lim _{s \rightarrow 0} \phi_{k l}(s)=0,
$$

which vanishes here for all $k$ and $l$. This result could be explained by the absence of real "dissipation" effects in harmonic systems like thermal conduction [12]. Additionally, due to the discontinuities in the time derivative of the autocorrelation function (8), we expect that the memory kernels contain several Dirac delta functions, as we have also seen numerically [8]:

$$
\begin{aligned}
\Phi_{k l}^{*}\left(t^{*}\right)= & -\delta\left(t^{*}-|k-l|+1\right)-\delta\left(t^{*}-|k-l|-1\right) \\
& +2 \delta\left(t^{*}-|k-l|\right)+\varphi_{k l}^{*}\left(t^{*}\right),
\end{aligned}
$$

where $\varphi_{k l}^{*}\left(t^{*}\right)$ is an otherwise smooth function of time. The discontinuities arise at the mesoscopic times $t^{*}=t / n$ that the wave fronts of the perturbed coarse-grained particle (containing $n$ oscillators, with $n \rightarrow \infty$ ) reach the boundaries of the coarse-grained particles as a consequence of the sound propagation. In principle, we could obtain $\varphi_{k l}^{*}\left(t^{*}\right)$ by calculating the inverse Laplace transform of (10). However, this is cumbersome and we actually prefer to calculate them numerically from molecular dynamics simulations, which also serves as a way to check the calculations. We present in Fig. 2 the first five memory kernels computed from a simulation with groups of $n=10000$ oscillators. The highly oscillatory raw data has been coarse grained in time by applying the time convolution $\Phi(t) \rightarrow \int d \tau \Phi(\tau) \theta(t-\tau)$, where $\theta(t)$ $=2 g(2 t / \Delta) / \Delta$, with $\Delta^{*}=\Delta / n=0.01$ [8]. The numerical Laplace transform of these kernels is in complete agreement with the analytical prediction given by (10). We can observe that the kernels are very slow decaying, having a significant magnitude at the end of the plot. In fact, we can use (10) to show that the absolute value of the kernel $\left|\varphi_{k l}^{*}\left(t^{*}\right)\right|$ is nonin- 


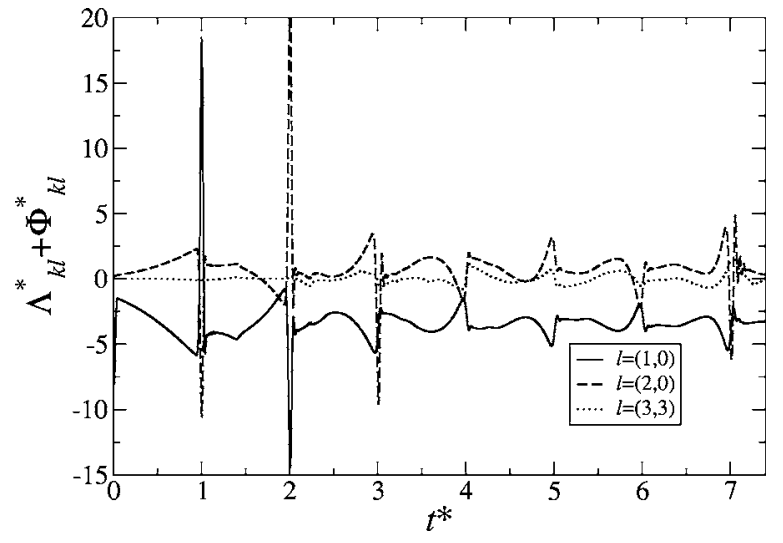

FIG. 3. Full memory kernels $\Phi_{k l}^{*}\left(t^{*}\right)$ oscillating around the conservative force values $\Lambda_{k l}^{*}$ in two dimensions. For a fixed oscillator $k$ chosen at the origin of the grid $(0,0)$, the various curves correspond to different oscillators $l$ along the grid.

tegrable. This is a consequence of the fact that the analytic continuation of $\phi_{k l}(s)$ presents an infinite number of singularities on the imaginary axis $(s=i \omega$ with real $\omega)$.

We have calculated numerically the memory kernels in higher dimensions. Figure 3 shows the first memory kernels of a two-dimensional system with square clumps containing $(400)^{2}$ oscillators. The data have been smoothed out with a mesoscopic time step of $\Delta^{*}=0.05$. The behavior of the kernels is very similar to that of the one-dimensional case. The first two kernels (solid and dashed lines) display the same Dirac deltas (conditioned in the figure by the convolution time step $\Delta^{*}$ ), as predicted by Eq. (14) in the onedimensional case. Additionally, we present in Fig. 4 the results of a three-dimensional system with cubic clumps containing $(95)^{3}$ oscillators and $\Delta^{*}=0.1$. Because the length of the clumps is too small to allow for a smooth representation of the kernels, we have also plotted in Fig. 4 the analogous results (dotted and dash-dotted lines) of a one-dimensional system with a similar clump size of 100 oscillators per group and $\Delta^{*}=0.1$. The behavior of the kernels remains similar.

The presence of the memory kernels, and the corresponding colored noise forces [13], would render the simulation of

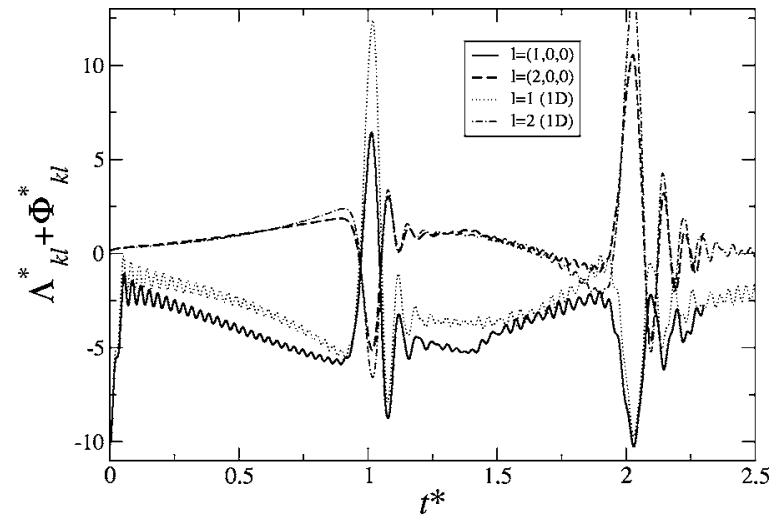

FIG. 4. The same system as in Fig. 3, but in three dimensions. The dotted and the dash-dotted lines are the corresponding functions of a 1-D system, plotted here as a reference.

the dissipative particles dynamics impractical due to computer memory limitations. A more promising approach would be to add new internal variables to the description so the process becomes Markovian. However, it is not clear if this can be done using a finite number of new internal variables. The thermodynamic variables (local density and internal energy) used in Refs. [4,5] would not account for the internal memory effect we have described in this work. This can be seen by considering a velocity perturbation traveling inside the coarse-grained particles. The center of mass, the local volume, and the internal energy of the coarse-grained particle would only be affected by the perturbation when it reaches the boundaries of the particle. On the other hand, a particle-based method could still be used if we regard them as virtual particles carrying energy, instead of representing portions of the system. In any case, the formalism used or the assumptions made would need to be closely related or checked at the microscopic level.

D. C. thanks J. Casado-Pascual for helpful discussions. This work has been partially supported by a grant from the European Union and the EPSRC.
[1] P. Hoogerbrugge and J. Koelman, Europhys. Lett. 19, 155 (1992).

[2] P. Español and P. Warren, Europhys. Lett. 30, 191 (1995).

[3] P. Español and M. Serrano, Phys. Rev. E 59, 6340 (1999).

[4] E. G. Flekkøy and P. V. Coveney, Phys. Rev. Lett. 83, 1775 (1999).

[5] P. Español, M. Serrano, and H. C. Öttinger, Phys. Rev. Lett. 83, 4542 (1999).

[6] P. Prinsen, P. Warren, and M. Michels, Phys. Rev. Lett. 89, 148302 (2002).

[7] M. Burke, R. Woscholski, and S. N. Yaliraki, Proc. Natl. Acad. Sci. U.S.A. 100, 13928 (2003).

[8] D. Cubero and S. N. Yaliraki, J. Chem. Phys. 122, 034108
(2005).

[9] R. Zwanzig, Nonequilibrium Statistical Mechanics (Oxford University Press, New York, 2001).

[10] N. W. Ashcroft and N. D. Mermin, Solid State Physics (Saunders, Fort Worth, 1976).

[11] R. Bird, C. F. Curtiss, R. Armstrong, and O. Hassager, Dynamics of Polymeric Liquids, Vol. 2: Kinetic Theory (Wiley, New York, 1987).

[12] G. Klein and I. Prigogine, Physica (Amsterdam) 19, 89 (1953).

[13] P. Hänggi and P. Jung, Adv. Chem. Phys. 89, 239 (1995).

[14] This force differs from that computed in P. Español, Phys. Rev. E 53, 1572 (1996) due to errors in the calculations. 